\title{
Avaliação do centro de gravidade de crianças e adolescentes com
}

\section{Mucopolissacaridose tipo VI}

\author{
Avaluation of center of gravity of children and adolescents with Mucopolysacaridosis type VI \\ Evaluación del centro de gravedad para niños y adolescentes con Mucopolisacaridosis tipo VI
}

Recebido: 16/02/2021 | Revisado: 22/02/2021 | Aceito: 23/02/2021 | Publicado: 03/03/2021

\author{
Sabrina de Melo Oliveira \\ ORCID: https://orcid.org/0000-0003-1024-4511 \\ Universidade Tiradentes, Brasil \\ E-mail: Sabrina.brml@gmail.com \\ Vanessa Lima \\ ORCID: https://orcid.org/0000-0002-5447-5330 \\ Universidade Tiradentes, Brasil \\ E-mail: nessa_eq@yahoo.com.br \\ Breno Azevedo \\ ORCID: https://orcid.org/0000-0003-4917-8726 \\ Universidade Tiradentes, Brasil \\ E-mail: brenodiazev@gmail.com \\ Bárbara Bernardo Figueirêdo \\ ORCID: https://orcid.org/0000-0002-4949-6268 \\ Universidade Tiradentes, Brasil \\ E-mail: barbara_bernardo@hotmail.com
}

\begin{abstract}
Resumo
Introdução: A mucopolissacaridose tipo VI (MPS VI) ou síndrome de Maroteaux-Lamy é uma doença metabólica rara, hereditária, conhecida como uma doença de depósito lisossomal onde os pacientes apresentam deformidades progressivas. Há uma necessidade de se entender como se comporta o centro de gravidade (CG) desses pacientes, como uma forma de prevenir quedas e direcionar a terapêutica. Objetivo: Avaliar a projeção do centro de gravidade de forma estática de crianças e adolescentes com MPS VI através do software de avaliação postural $\left(\mathrm{SAPO}^{\circledR}\right)$. Metodologia: Trata-se de um estudo descritivo, do tipo corte transversal, realizado em um centro de referência em doenças raras, com pacientes com MPS VI. A avaliação foi realizada de forma estática através de analise utilizando o SAPO $^{\circledR}$. Resultados: Foram avaliados 15 voluntários, $60 \%$ (9) do sexo masculino, com idade média de 7,80 \pm 3,66. Foram encontradas assimetrias nos planos frontal e sagital, sendo uma maior assimetria no plano sagital: $34,68 \% \pm 18,04$ de assimetria, apresentando valor máximo de 73,7\%. O CG de 93,9\% dos avaliados estavam anteriorizados. Conclusão: Baseando-se nos relatórios emitidos pelo SAPO ${ }^{\circledR}$, pode-se afirmar que existem alterações no CG das crianças e adolescentes com MPS VI.
\end{abstract}

Palavras-chave: Mucopolissacaridose VI; Criança; Adolescente; Equilíbrio postural; Fisioterapia.

\begin{abstract}
Introduction: Mucopolysaccharidosis type VI (MPS VI) or Maroteaux-Lamy syndrome is a rare, hereditary metabolic disease, known as a lysosomal deposit disease where patients have progressive deformities. There is a need to understand how the center of gravity (CG) of these patients behaves, as a way of preventing falls and directing therapy. Objective: To evaluate the projection of the center of gravity in a static way for children and adolescents with MPS VI through the postural assessment software (SAPO®). Methodology: This is a descriptive, cross-sectional study, carried out in a reference center for rare diseases, with patients with MPS VI. The evaluation was performed in a static way through analysis using SAPO ${ }^{\circledR}$. Results: 15 volunteers were evaluated, $60 \%$ (9) were male, with an average age of $7.80 \pm 3.66$. Asymmetries were found in the frontal and sagittal planes, with a greater asymmetry in the sagittal plane: $34.68 \% \pm 18.04$ asymmetry, with a maximum value of $73.7 \%$. The CG of $93.9 \%$ of those evaluated were anteriorized. Conclusion: Based on the reports issued by $\mathrm{SAPO}{ }^{\circledR}$, it can be said that there are changes in the CG of children and adolescents with MPS VI.
\end{abstract}

Keywords: Mucopolysaccharidosis VI; Child; Adolescent; Postural balance; Physical therapy specialty.

\section{Resumen}

Introducción: La mucopolisacaridosis tipo VI (MPS VI) o síndrome de Maroteaux-Lamy es una enfermedad metabólica hereditaria poco frecuente, conocida como enfermedad de depósito lisosómico en la que los pacientes presentan deformidades progresivas. Es necesario comprender cómo se comporta el centro de gravedad (GC) de estos 
pacientes, como una forma de prevenir caídas y dirigir la terapia. Objetivo: Evaluar la proyección del centro de gravedad de forma estática para niños y adolescentes con MPS VI a través del software de evaluación postural (SAPO®). Metodología: Se trata de un estudio descriptivo, transversal, realizado en un centro de referencia de enfermedades raras, con pacientes con MPS VI. La evaluación se realizó de forma estática mediante análisis mediante SAPO®. Resultados: se evaluaron 15 voluntarios, 60\% (9) varones, con una edad promedio de 7,80 \pm 3,66. Se encontraron asimetrías en los planos frontal y sagital, con mayor asimetría en el plano sagital: 34,68\% \pm 18,04 asimetría, con un valor máximo de 73,7\%. El GC del 93,9\% de los evaluados fue anteriorizado. Conclusión: Con base en los informes emitidos por SAPO®, se puede decir que existen cambios en el GC de niños y adolescentes con MPS VI.

Palabras clave: Mucopolisacaridosis VI; Niño; Adolescente; Equilibrio postural; Fisioterapia.

\section{Introdução}

A mucopolissacaridose tipo VI (MPS VI) ou síndrome de Maroteaux-Lamy é uma doença metabólica rara, hereditária, conhecida como uma doença de depósito lisossomal (Akyol et al. 2019; Entchev et al. 2020). Causada pela deficiência da enzima lisossômica $\mathrm{N}$-acetilgalactosamina-4-sulfatase ou arilsufatase B, responsável pela degradação dos glicosaminoglicanos (GAG) sulfato de dermatina e sulfato de condroitina (Turtelli, 2002; Pereira, Garbeli \& Palazzo et al. 2011; Medeiros, Silva, Queiroz, Cattuzzo \& Soares, 2015).

Como os GAG não são degradados devido a enzima deficitária, seu acúmulo pode levar a várias deformidades multissistêmicas progressivas, que se intensificam na medida que ocorre mais acúmulo, gerando limitações de movimentos, rigidez articular e contraturas flexionais das articulações (Pereira et al. 2011; Medeiros et al. 2015; Figueirêdo, Magalhães, Andrade, Bezerra \& Duarte, 2018).

Além disso, são encontradas gibosidades, encurtamento dos membros e do pescoço, déficit de estatura, cifose tóracolombar, inclinação do tronco e da pelve, flexão de quadril e joelho, pé equino, além do comprometimento da articulação do ombro, o que pode causar uma desordem no movimento (balance) e essas deformidades podem comprometer a marcha e o equilíbrio nessa população (Medeiros et al. 2015; Khan, 2017; Anjos, Figueirêdo \& Cavalcanti, 2018).

O comprometimento do equilíbrio estático e dinâmico, com risco de queda importante foi encontrado em estudo anterior realizado com pacientes com a MPS VI (Anjos et al. 2018). O risco de queda nesta população pode vir a debilitá-los ainda mais. Esses achados devem estar relacionados às alterações do centro de gravidade (CG), visto que estas alterações estão relacionadas com diversas características físicas individuais, como estatura, percentual de massa muscular, distribuição de massa corporal, comprimento dos segmentos corporais, entre outras (Narciso et al. 2010; Entchev et al. 2020).

O CG é conceituado como um lugar geométrico no qual se concentra todas as massas do corpo, ponto onde o mesmo se equilibra de acordo com a aceleração e gravidade local, distribuindo de forma homogênea o peso e posicionando o corpo de maneira estável (Braz, Goes \& Carvalho, 2008; Narciso et al. 2010), este parâmetro tem sido estudado há décadas, como forma de instrumento de diagnóstico, planejamento e de acompanhamento do tratamento, inclusive fisioterapêutico, podendo ser avaliado quantitativamente através da interpretação de fotografias que permitem a aquisição de valores que definem o alinhamento corporal e passam registros sobre as assimetrias no plano frontal e sagital do CG. (Braz et al. 2008; Lemos, Teixeira \& Mota, 2009).

O CG pode ser estudado através de softwares específicos para o cálculo de ângulos, distâncias verticais e horizontais. Um software bastante utilizado na área da saúde é o software de avaliação postural (SAPO ${ }^{\circledR}$ ), por ser de acesso livre e gratuito, além de ser uma ferramenta válida e confiável para a aquisição de medidas e ângulos de segmentos (Braz et al. 2008; Costa, Gonçalves, Carvalho, Ferreira, \& Cunha, 2018).

Estudos que avaliam o CG dessa população são escassos, não sendo encontrados até o presente momento na literatura pesquisada. A escassez é ainda maior se tratando de um subtipo que é considerado um dos mais raros no mundo, a MPS VI 
(Mizuno et al. 2010). Sendo assim, há uma necessidade de se entender como se comporta o centro de gravidade desses pacientes, como uma forma de prevenir quedas, direcionar a terapêutica, e trabalhar precocemente nesse grupo específico de doenças graves e raras. Por isso, o presente estudo teve como objetivo avaliar a projeção do centro de gravidade de forma estática de crianças e adolescentes com MPS VI através do SAPO ${ }^{\circledR}$.

\section{Metodologia}

Trata-se de um estudo descritivo, do tipo corte transversal e com uma abordagem qualitativa. O estudo foi realizado no Centro de Referência em Erros Inatos do Metabolismo do Instituto de Medicina Integral Professor Fernando Figueira (CETREIM-IMIP), localizado em Recife/PE, sob aprovação do Comitê de Ética em Humanos do IMIP (nº 4713-15).

A amostra foi constituída por indivíduos com MPS VI, de ambos os sexos, acima de dois anos de idade. Todos os participantes estavam cadastrados na Associação Pernambucana de Mucopolissacaridoses (AMPS-PE), e possuíam a Mucopolissacaridose tipo VI comprovada por dosagem enzimática via amostra sanguínea.

Pacientes diagnosticados em prontuário com déficit cognitivo, alterações neurológicas e alterações auditivas que comprometesse as variáveis da pesquisa, e os que não poderiam ficar na postura ortostática de forma independente foram excluídos do estudo.

Após a verificação do preenchimento dos critérios de inclusão, os pesquisadores convidaram todos os indivíduos e seus responsáveis legais, para participarem da pesquisa, explicando os objetivos do estudo, e todas as dúvidas foram esclarecidas, foi assinado o Termo de Consentimento Livre e Esclarecido (TCLE) pelo responsável legal e os menores acima de 10 anos de idade, assinaram o Termo de Assentimento Livre e Esclarecido (TALE) dando o consentimento para a realização da pesquisa. Os analfabetos ou os impossibilitados de assinar deram o consentimento através do registro da impressão digital.

A coleta de dados respeitou o agendamento das consultas e terapias de rotina, as avaliações do centro de gravidade foram realizadas em uma sala reservada no CETREIM-IMIP. Foram coletadas informações pessoais dos pacientes, a fim de obter seus dados sociodemográficos, clínico-biológicos a partir de um formulário elaborado pelos próprios pesquisadores. Todos foram avaliados de forma individual, com a participação dos seus responsáveis quando era o caso.

A avaliação foi realizada de forma estática por meio de registro fotográfico e análise através do $\mathrm{SAPO}^{\circledR}$, as fotos foram calibradas de acordo com um referencial fixo constituído por um fio de prumo com a marcação de um metro, marcadores esféricos foram acoplados à pele, em pontos anatômicos de referência do software, foram realizadas medições de distâncias e de ângulos corporais. Durante a avaliação, os participantes foram instruídos a adotar a posição em pé, de olhos abertos, com os pés em paralelo, porém de forma livre, em sua postura estática habitual em espaço calmo e reservado.

Foi utilizada uma câmera fotográfica digital (Sony® DSC-HX60 de 20.4 megapixels) posicionada em um tripé, há 2 (dois) metros dos participantes. Quatro imagens foram capturadas: vista anterior, vista posterior, lateral direita e lateral esquerda do corpo (figura 1). A coleta de dados incluindo desde a explicação da pesquisa e assinatura dos termos a colocações dos marcadores e captação das imagens durou em média 40 minutos. A coleta de dados teve a duração de dois meses respeitando os dias de atendimento dos pacientes ao CETREIM-IMIP.

As fotos foram obtidas com os participantes minimamente vestidos, de modo a detectar referências ósseas anatômicas pré-determinadas. Todos os marcadores foram colocados bilateralmente, à exceção de C7 e T3 (Figura 2).

O CG foi calculado através da estimativa da sua posição a partir das imagens, utilizando parâmetros antropométricos, pontos anatômicos de acordo com a relação entre a projeção do CG e a assimetria do plano frontal e sagital. Foram calculadas as assimetrias no plano frontal e sagital que mostram a posição do CG de cada voluntário em relação aos maléolos laterais, 
através de expressões em porcentagem de cada assimetria. Além disso, foi observada pelas fotografias a presença visível de escolioses e descritas em prontuário como: escoliose visível presente ou ausente.

Foi avaliado o ponto de aplicação da força gravitacional resultante sobre o corpo que age sobre a base de suporte, área delimitada pelas bordas laterais dos pés. Essa base de suporte fornece um limiar de estabilidade, na qual podem ser realizadas diversas tarefas e movimentos sem que ocorra a perda do equilíbrio, expressando, assim, a base de suporte funcional do indivíduo.

Figura 1. Posicionamento do tripé, câmera e voluntário no momento de coleta das fotografias nas vistas anterior, posterior, lateral direita e lateral esquerda de acordo com as marcações das referências ósseas do protocolo do SAPO ${ }^{\circledR}$.

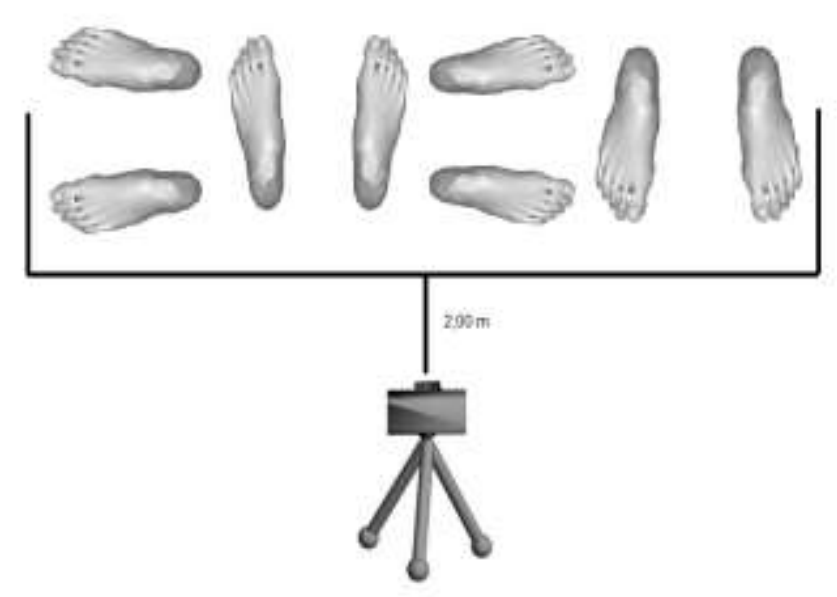

Fonte: Autores (2021).

Figura 2. Pontos de referência utilizados de acordo com as marcações das referências ósseas do protocolo do SAPO ${ }^{\circledR}$.

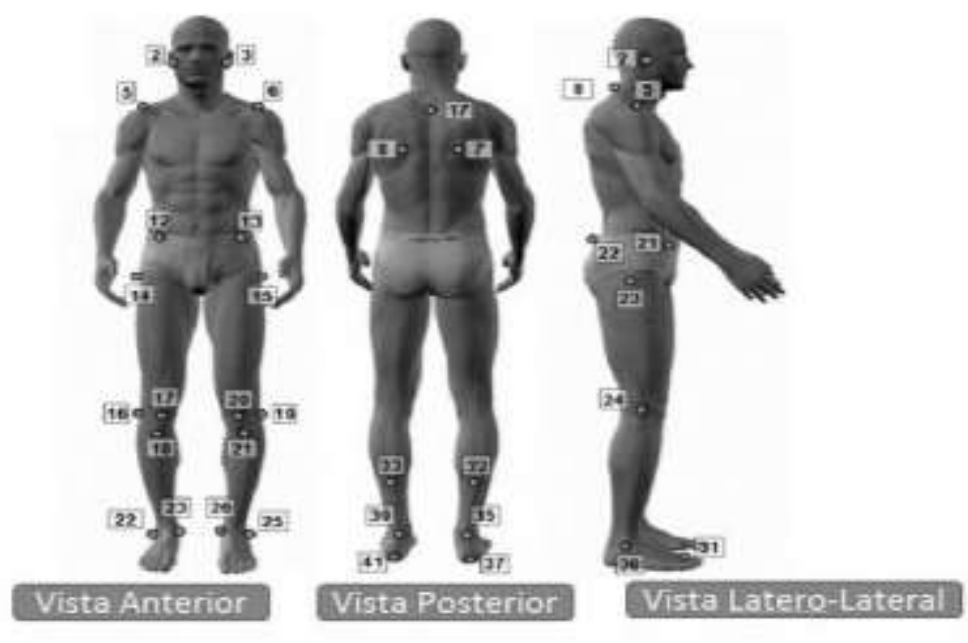

Fonte: $\mathrm{SAPO}^{\circledR}$

$\mathrm{Na}$ vista anterior (2, 3: tragus direito e esquerdo; 5, 6: acrômio direito e esquerdo; 12, 13: espinha ilíaca ânterosuperior direita e esquerda; 14, 15: trocânter maior direito e esquerdo; 16, 19: projeção lateral da linha articular do joelho 
direito e esquerdo; 17, 20: centro da patela direita e esquerda; 18, 21: tuberosidade da tíbia direita e esquerda; 22, 25: maléolos laterais; 23, 26 maléolos mediais).

Na vista posterior (7, 8: ângulo inferior da escápula direita e esquerda; 17 terceira vértebra torácica; 32, 33: ponto medial da perna, 35, 39: linha intermaleolar; 37, 41 tendão calcâneo bilateralmente). Na vista lateral (2: tragus; 8: sétima vértebra cervical; 5: acrômio; 21: espinha ilíaca antero-superior; 22: espinha ilíaca póstero-superior; 23 trocânter maior; 24 : projeção da linha articular do joelho; 30: maléolo lateral; 31 região entre o segundo e o terceiro metatarso.

Os dados coletados foram submetidos à uma análise descritiva com valores absolutos e relativos (média, desvio padrão e porcentagem). A pesquisa não acarretou danos aos participantes, apenas um possível constrangimento por precisar estar com o mínimo de roupa possível para a avaliação.

\section{Resultados}

Foram abordados 16 voluntários, porém, participaram do estudo 15 pacientes, visto que um dos entrevistados foi excluído por não conseguir manter-se na postura ortostática estática, que é necessária para a captura da imagem e análise pelo SAPO, ocasionando na impossibilidade do software analisar os dados do paciente, sendo assim o mesmo teve seu resultado anulado pelo SAPO. Dos 15 avaliados, 9 (60\%) eram do sexo masculino, com idade média de 7,80 \pm 3,66 e com média do tempo de diagnóstico da MPS de 58,74 \pm 25,21 meses. Todos faziam terapia de reposição enzimática (TRE) semanalmente, possuíam peso médio de $20,1 \pm 5,2 \mathrm{~kg}$, altura média de $1,08 \pm 0,12 \mathrm{~cm}$ e média do índice de massa corpórea foi de $18,6 \pm 2,6$ $\mathrm{kg} / \mathrm{m} 2$.

Dentre eles $4(26,7 \%)$ realizavam a fisioterapia uma vez na semana, 9 (60\%) residiam na região metropolitana do Recife e 6 (40\%) na zona rural do estado de Pernambuco. Nenhum paciente fazia uso de órteses de correção ou de posicionamento, (12) $80 \%$ apresentaram dores articulares nos MMII, principalmente em joelhos 9 (60\%) e a escoliose estava presente em $6(40 \%)$ dos indivíduos avaliados.

Foi encontrada a cor parda em 8 (53,3\%) dos voluntários, cor negra 3 (20\%) cor branca 3 (20\%) e glaucoma diagnosticado em 10 (66,6\%). Dificuldades em equilibra-se em um pé só (apoio unipodal de olhos abertos) 14 (93,33\%). Dificuldades em equilibrar-se em apoio bipodal com os olhos alternadamente abertos e fechados durante 10 segundos (9 pacientes, $60 \%)$.

A análise através do $\mathrm{SAPO}^{\circledR}$ foi realizada no plano frontal e no plano sagital. No plano frontal mostrou uma média de assimetria de 3,66\% e desvio padrão de \pm 15,84, apresentando valor máximo de 34,4\%. O plano sagital mostrou uma média de assimetria de $34,68 \%$ e desvio padrão de $\pm 18,04$, apresentando valor máximo de 73,7\%. Durante o cálculo da assimetria do plano sagital, pelo fato do software não ter fornecido dados de um dos pacientes, a média das assimetrias desse plano foi realizada com os dados de 14 pacientes.

Observou-se que $14(93,9 \%)$ pacientes estavam anteriorizados, quanto a análise de assimetria do plano frontal e sagital (látero-lateral), mostrou que $8(53,3 \%)$ foi para direita, $5(33,3 \%)$ para a esquerda, $1(6,7 \%)$ ficou centralizado e 1 $(6,7 \%)$ não apresentou assimetria no plano sagital. 
Quadro 1. Resultados das assimetrias mensuradas expressas em porcentagem.

\begin{tabular}{|c|c|c|}
\hline $\begin{array}{l}\text { VOLUNTÁRIOS DO } \\
\text { ESTUDO }\end{array}$ & $\begin{array}{l}\text { ASSIMETRIA PLANO } \\
\text { FRONTAL }(\%)\end{array}$ & $\begin{array}{l}\text { ASSIMETRIA PLANO } \\
\text { SAGITAL }(\%)\end{array}$ \\
\hline PACIENTE 1 & $2.5 \%$ & $29.7 \%$ \\
\hline PACIENTE 2 & $19.8 \%$ & $73.7 \%$ \\
\hline PACIENTE 3 & $6.7 \%$ & - \\
\hline PACIENTE 4 & $3.7 \%$ & $13.2 \%$ \\
\hline PACIENTE 5 & $-8.0 \%$ & $54.6 \%$ \\
\hline PACIENTE 6 & $10.9 \%$ & $25.5 \%$ \\
\hline PACIENTE 7 & $6.1 \%$ & $55.9 \%$ \\
\hline PACIENTE 8 & $0.7 \%$ & $30.2 \%$ \\
\hline PACIENTE 9 & $16.7 \%$ & $26.7 \%$ \\
\hline PACIENTE 10 & $-11.9 \%$ & $36.4 \%$ \\
\hline PACIENTE 11 & $-19.2 \%$ & $37.2 \%$ \\
\hline PACIENTE 12 & $4.8 \%$ & $22.2 \%$ \\
\hline PACIENTE 13 & $-28.1 \%$ & $29.3 \%$ \\
\hline PACIENTE 14 & $15.8 \%$ & $43.8 \%$ \\
\hline PACIENTE 15 & $34.4 \%$ & $41.9 \%$ \\
\hline & Média: $3,66 \% \pm 15,84$ & Média: $34,68 \% \pm 18,04$ \\
\hline
\end{tabular}

* -: Não foi gerado dado pelo software. Fonte: dados da pesquisa.

Figura 3. Projeção do centro de gravidade em crianças e adolescentes com MPS VI $(n=14)$.

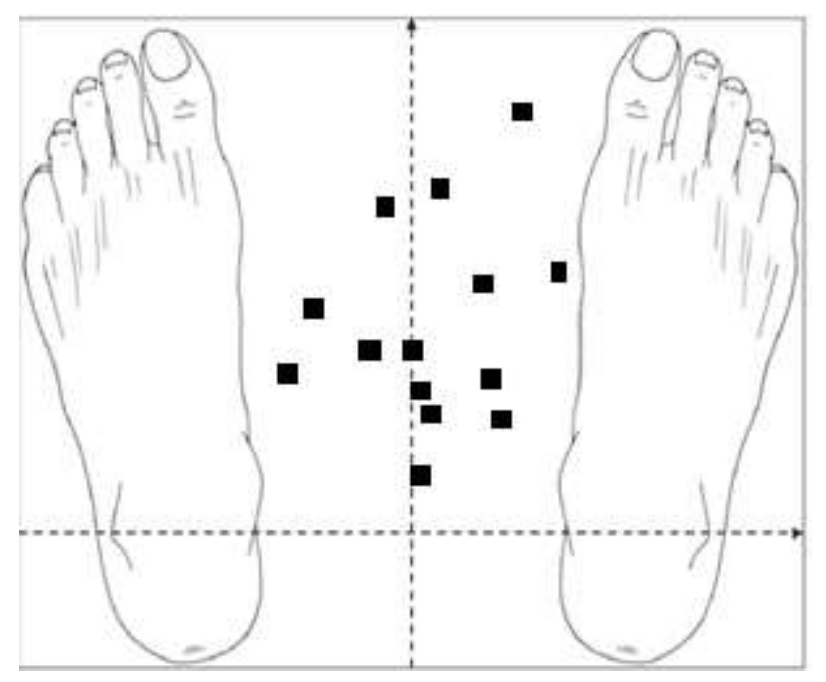

Fonte: dados da pesquisa.

\section{Discussão}

Até o momento não foi encontrado outro estudo que tenha avaliado a projeção do CG de crianças e adolescente diagnosticados com MPS VI, através do SAPO ${ }^{\circledR}$. Este estudo parece ser pioneiro na investigação do CG desse grupo. As 
assimetrias encontradas já eram esperadas visto que na MPS VI são encontradas diversas alterações do sistema osteoarticular, a doença caracteriza-se por uma ampla gama de apresentações clínicas, um estudo de 2008, observou vinte e nove pacientes com MPS tipo VI no estudo, desses $60 \%$ referiram alterações ósseas como primeiro sintoma e a faixa de aparecimento dos sintomas foi de 0 a 24 meses e o diagnóstico ocorreu entre 24 a 96 meses (Vieira et al. 2008).

Na MPS tipo VI ocorrem mutações no gene Arilsulfatase B do cromossomo 5q13-14, que codifica a enzima e a diversidade das mutações provavelmente é responsável pela grande heterogeneidade clínica da doença (Herskhovitz et al. 1999). Este fato pode explicar a razão por assimetrias heterogêneas, com variações amplas, explicaria o caso de indivíduos com assimetrias graves e outros apenas com pequenas assimetrias.

Além disso, a população pediátrica tem particularidades em relação ao equilíbrio e consequentemente ao CG, pois ocorrem muitas transformações na postura, por exemplo: no período de 7 a 12 anos de idade, ocorrem transformações na postura em direção a um novo equilíbrio compatível com novas proporções corporais (Santos, Silva, Sanada \& Alves, 2009). Deformidades musculoesqueléticas associadas ao desalinhamento de tronco prejudicam o desenvolvimento da criança, acarretam alterações na marcha e até mesmo a funcionalidade na postura sentada (Holmes, Michael, Thorpe \& Solomonidis, 2003; Rocha et al. 2019; Silva et al. 2020).

Durante a avaliação, foi encontrada anteriorização e lateralização do CG nos participantes. Estes achados podem ser justificados visto que pacientes com MPS, desde os primeiros anos de vida apresentam comumente a restrição da amplitude de movimentação, principalmente dos joelhos, fazendo com que os pacientes assumam uma postura com joelhos flexionados, além disso, a hepatoesplenomegalia e o aumento dos órgãos internos, podem gerar abdômen protuso, contribuindo para a alteração do centro de gravidade, como a anteriorização (Cardoso-Santos et al. 2008; Mizuno et al. 2010; Valayannopoulos, Nicely \& Harmatz, 2010).

O fato do abdômen se projetar para frente acentua o arqueamento das costas e provoca hiperlordose (Brukner \& Khan, 2001), deformidade encontrada nos pacientes com MPS VI (Turtelli, 2002, Anjos et al. 2018). Em um indivíduo em posição anatômica, ereta e estática, o centro de gravidade encontra-se ligeiramente anterior à segunda vértebra sacral, próximo aos quadris e aproximadamente, a 55\% da sua estatura (Enoka, 2000; Narciso et al. 2010), essa referência é padronizada para o grupo de indivíduos adultos. Dados quantitativos sobre o alinhamento postural de crianças sadias em desenvolvimento são escassos, e os valores de referência para os desvios são baseados na postura da população adulta.

No entanto, é sabido que o sistema musculoesquelético em desenvolvimento detém características próprias e apresenta alinhamentos posturais transitórios considerados anormais nos adultos (Santos et al. 2009). Segundo Calvete (2004), os indivíduos obesos também têm alterações estruturais como, por exemplo, apresentam com frequência abdômen protuso e, consequentemente, tem o CG deslocado anteriormente, uma hiperlordose lombar e uma anteversão pélvica.

Em um estudo anterior realizado, Dhawale et al. (2012), afirmam que os pacientes com MPS VI apresentam uma maior inclinação do tronco e da pelve para frente ao caminhar, também um aumento da flexão de quadril e joelho, diminuição da dorsiflexão do tornozelo, rotação externa de quadril, aumento da adução do quadril, genu valgo e torção tibial externa.

Estes pacientes geralmente caminham lentamente e com passos curtos. Narciso et al. (2010), afirmaram que as alterações do CG estão relacionadas com diversas características físicas individuais, como estatura, percentual de massa muscular, distribuição de massa corporal, comprimento dos segmentos corporais, entre outros.

Para manterem-se em equilíbrio os pacientes com MPS acabam projetando seu CG para a região do ante-pé, devido a anteroversão da pelve que já foi encontrada em estudo realizado por Santos et al. (2012), envolvendo pacientes com escoliose, alteração também encontrada em indivíduos com MPS VI (Valayannopoulos et al. 2010). No nosso estudo a escoliose visível estava presente em $6(40 \%)$ dos avaliados, este fato pode justificar também as assimetrias encontradas. 
Desta forma, os pacientes com MPS VI apresentam redução da base de apoio devido a anteriorização e lateralização do CG e é na região do ante-pé onde muitos dos pacientes conseguem manter o equilíbrio devido a postura de flexão dos joelhos e do quadril associada as deformidades esqueléticas já citadas anteriormente (Dhawale et al. 2012; Anjos et al. 2018). Bases de apoio reduzidas aumentam a dificuldade de estabilização, o que exige maior estabilidade de ligamentos, músculos e articulações para que a manutenção do equilíbrio seja mantida (Dorneles, Pranke, Lemos \& Teixeira, 2014).

Anjos et al. (2018), constataram diferenças nas mensurações do comprimento dos membros de pacientes com MPS VI, desalinhamento dos segmentos esqueléticos e que a baixa estatura é um dos primeiros sinais da MPS VI. Devido ao desenvolvimento anormal dos órgãos viscerais, os pacientes com MPS VI apresentam uma grande circunferência abdominal. Pessoas com protrusão de abdômen tem maior dificuldade para iniciar a marcha e manter o equilíbrio, devido a mudança da relação antropométrica-anatômica e da mudança na altura do centro de gravidade (Anjos et al. 2018).

No estudo de Aspdena, Rudmana e Meakin, (2006) foi realizada uma análise do funcionamento cinesiológico da articulação do quadril, a qual segundo os autores é o mecanismo de equilíbrio postural do tronco dos seres humanos, estando o CG geralmente equilibrado acima dos quadris. O CG de uma pessoa na posição ortostática estática e com a coluna ereta se encontra a cerca de 3,0 cm na frente da articulação do tornozelo sobre uma linha vertical que toca o chão, quando estão posicionados com braços ao longo do corpo (Costa et al. 2018).

Fonseca e Scheicher (2012), encontraram uma correlação entre os escores da Escala de Equilíbrio de Berg (EBB) e as assimetrias sagital e frontal do CG, indicando que quanto mais anteriorizado/lateralizado for o centro de gravidade, menor o equilíbrio, sugerindo um maior índice de quedas. Anjos et al. (2018), observaram um comprometimento do equilíbrio nos pacientes com MPS VI e um grande risco de quedas. Lemos et al. (2009), referiram possíveis relações entre a posição do centro de gravidade com a manutenção do equilíbrio estático e/ou postura corporal.

As crianças com MPS estudadas tendenciavam a apresentar flexão dos joelhos e leve elevação dos calcanhares, assim como nas crianças estudadas por Avelar, Silva, Adriano e Vieira (2006), que se utilizaram do mesmo procedimento para a manutenção do equilíbrio quando na posição ortostática estática. Em razão da clínica da MPS VI ser muito vasta, é importante a avaliação do CG e o acompanhamento por equipe multidisciplinar, para prevenir, diagnosticar e tratar precocemente as consequências dessas alterações encontradas neste estudo.

As assimetrias do centro de gravidade devem ser trabalhadas por programas de fisioterapia centrados em corrigir/minimizar deformidades, alterações posturais e encurtamentos musculares, assim como ganhar amplitude de movimento e realizar treinos proprioceptivos, focadas em melhorar o equilíbrio e consequentemente a marcha desses pacientes. Este estudo teve como limitação o número reduzido de sujeitos e a impossibilidade da realização de correlações, a subjetividade em diagnosticar a presença da escoliose e a dificuldade em manter as crianças em postura estática.

\section{Considerações Finais}

Crianças e adolescentes com MPS VI apresentaram alterações do centro de gravidade nos planos frontal e sagital, sendo uma maior assimetria no plano sagital, e a maioria dos avaliados apresentaram uma anteriorização do CG. Se fazem necessários estudos mais abrangentes abordando as complicações que a alteração do centro de gravidade pode causar em pacientes com MPS VI e planos de tratamento para minimizá-las

\section{Referências}

Akyol, M. U., Alden, T. D., Amartino, H., Ashworth, J., Belani, K., Berger, K. I., Borgo, A., Braunlin, E., Eto, Y., Gold, J. I., Jester, A., Jones, S. A., Karsli, C., Mackenzie, W., Marinho, D. R., McFadyen, A., McGill, J., Mitchell, J. J., Muenzer, J., \& Okuyama, T. MPS Consensus Programme Co-Chairs (2019). Recommendations for the management of MPS VI: systematic evidence- and consensus-based guidance. Orphanet journal of rare diseases, $14(1)$, 118. https://doi.org/10.1186/s13023-019-1080-y 
Anjos, N. H. L. R., Figueirêdo, B. B. R., Silva \& Cavalcanti, D. B. A. (2018). Avaliação do equilíbrio e risco de queda em pacientes com mucopolissacaridose VI. Fisioterapia Brasil, 19(4), 500-7.

Aspden, R. M., Rudman, K. E., \& Meakin, J. R. (2006). A mechanism for balancing the human body on the hips. Journal of biomechanics, 39(9), 1757-9. https://doi.org/10.1016/j.jbiomech.2005.04.028

Avelar, I. S., Silva, R. Q., Adriano, T. R., \& Vieira, M. F. (2006). Importância da biomecânica para o professor de educação física: observando uma brincadeira infantil. Pensar a Prática, 3, 106-10. https://doi.org/10.5216/rpp.v3i0.34

Braz, R., Goes, F., \& Carvalho, G. (2017). Confiabilidade e validade de medidas angulares por meio do software para avaliação postural. Fisioterapia em Movimento, 21(3). https://periodicos.pucpr.br/index.php/fisio/article/view/19185.

Brukner, P., \& Khan, K. (2001). Clinical sports medicine. (2a ed.): McGraw-Hill.

Cardoso-Santos, A. A., Ana C. M. M., Fagondes, S., Burin, M. G., Giugliani, R, \& Schwartz, I. V. D. (2008). Mucopolissacaridose tipo VI (síndrome de Maroteaux-Lamy): avaliação da mobilidade articular e das forças de garra e de pinça. Jornal de Pediatria, 84(2), 130-5. https://dx.doi.org/10.1590/S002175572008000200007.

Calvete, A., S. (2004). A relação entre alteração postural e lesões esportivas em crianças e adolescentes obesos. Motriz, 10(2), 67-72.

Costa, R, F., Gonçalves, N., R., Carvalho, S, M, P., Ferreira, G., \& Cunha, R, G. (2018). Caracterização da projeção do centro de gravidade de bailarinas com deficiência auditiva durante o ortostatismo. e-Scientia, Belo Horizonte, 11(1), 1-10.

Dhawale, A. A., Church, C., Henley, J., Holmes, L., Jr, Thacker, M. M., Mackenzie, W. G., \& Miller, F. (2013). Gait pattern and lower extremity alignment in children with Morquio syndrome. Journal of pediatric orthopedics. Part B, 22(1), 59-62. https://doi.org/10.1097/BPB.0b013e32835a0e6d.

Dorneles, P. P., Pranke, G. I., Lemos, L. F. C., \& Teixeira, C. (2014). Análise biomecânica relacionada a lesões no balé clássico. Revista Mackenzie de Educação Física e Esporte, 13(2).

Enoka, R. M. (2000). Bases neuromecânicas da cinesiologia. (2a ed.): Manole.

Entchev, E., Jantzen, I., Masson, P., Bocart, S., Bournique, B., Luccarini, J. M., Bouchot, A., Lacombe, O., Junien, J. L., Broqua, P., \& Tallandier, M. (2020). Odiparcil, a potential glycosaminoglycans clearance therapy in mucopolysaccharidosis VI-Evidence from in vitro and in vivo models. PloS one, 15(5), e0233032. https://doi.org/10.1371/journal.pone.0233032

Figueirêdo, B. B. R. S., Magalhães, P. A F., Andrade, L. B. D., Bezerra, P., \& Duarte, M. C. M. B (2018). Nível de independência, capacidade funcional e força muscular respiratória de pacientes com mucopolissacaridose tipo VI no Nordeste do Brasil. Revista Brasileira de Saúde Materno Infantil, 18(1), 83-92. https://doi.org/10.1590/1806-93042018000100004.

Fonseca, L. C. S., \& Scheicher M. E. (2012). Relação entre projeção do centro de gravidade e equilíbrio em idosos. Ter Man, 10, 440-3.

Herskhovitz, E., Young, E., Rainer, J., Hall, C. M., Lidchi, V., Chong, K., \& Vellodi, A. (1999). Bone marrow transplantation for Maroteaux-Lamy syndrome (MPS VI): long-term follow-up. Journal of inherited metabolic disease, 22(1), 50-62. https://doi.org/10.1023/a:1005447232027.

Holmes, K. J., Michael, S. M., Thorpe, S. L., \& Solomonidis, S. E. (2003). Management of scoliosis with special seating for the non-ambulant spastic cerebral palsy population--a biomechanical study. Clinical biomechanics (Bristol, Avon), 18(6), 480-7. https://doi.org/10.1016/s0268-0033(03)00075-5.

Khan, S. A., Peracha, H., Ballhausen, D., Wiesbauer, A., Rohrbach, M., Gautschi, M., Mason, R. W., Giugliani, R., Suzuki, Y., Orii, K. E., Orii, T., \& Tomatsu, S. (2017). Epidemiology of mucopolysaccharidoses. Molecular genetics and metabolism, 121(3), 227-40. https://doi.org/10.1016/j.ymgme.2017.05.016.

Lemos, L. F. C., Teixeira, C. S., \& Mota, C. B. (2009). Uma revisão sobre centro de gravidade e equilíbrio corporal. R. bras. Ci. e Mov., 17(4), 83-90.

Medeiros, J. N. S., Silva, B. B. R., Queiroz, D, R., Cattuzzo, M. R., \& Soares, K. M. S. (2015). Avaliação da força de preensão e amplitude de movimentos dos membros superiores em pacientes com mucopolissacaridose VI. Revista Acta Fisiátrica. Recife, 22(2). https://doi.org/10.5935/0104-7795.20150013.

Mizuno, C. A., Figueiredo, J. B., Teza, I. T. V., Taira, L. G. N., Silva, T. A., Paixão, D. L., \& Mizuno, J. C. (2010). Aspectos clínicos da mucopolissacaridose tipo VI. Rev. Bras. Clin. Med., 8. 356-61.

Narciso, F. V., Santos, S. S., Ferreira, F., Lemos, V. S, Barauna, M. A., Cheik, N. A., \& Canto, R. S T. (2010). Altura percentual do centro de gravidade e número de quedas em idosos ativos e sedentários. Revista Brasileira de Cineantropometria \& Desempenho Humano, 12(4), 302-7. https://doi.org/10.5007/1980-0037.2010V12N4P302.

Pereira, J. O., Garbeli, M. G. de A. M., \& Palazzo, V. C. (2011). Mucopolissacaridose tipo VI: evolução natural, importância diagnóstica e terapêutica. Revista Neurociências, 19(2), 329-38. https://doi.org/10.34024/rnc.2011.v19.8380.

Rocha, B. N., Costa, C. A. da., Lago, F. C., Aruda, J. M. P., de, Abreu, P. G., Schumacher, C., Kruel, C. S, Guazina, F. M. N., \& Carlesso, J. P. P (2019). Crianças no lugar público: contribuições para um desenvolvimento saudável. Pesquisa, Sociedade e Desenvolvimento , 8 (2), e1582595. https://doi.org/10.33448/rsd-v8i2.595

Silva, M. de O., Cunha, A. C. C. da., Soares, E. L., Santino, A. C. D., Silva, J. L. da., \& Ribeiro, A. da S. (2020). Chronic conditions in childhood: consequences for healthy brothers and Nurse's performance in family care. Research, Society and Development, 9(8), e241985650. https://doi.org/10.33448/rsd-v9i8.5650 
Research, Society and Development, v. 10, n. 3, e1810313036, 2021

(CC BY 4.0) | ISSN 2525-3409 | DOI: http://dx.doi.org/10.33448/rsd-v10i3.13036

Santos, L. M., Souza, T. P., Crescentini, M. C. T., Poletto, P. R., Gotfryd, A. O., \& Yi, L. C. (2012). Avaliação postural por fotogrametria em pacientes com escoliose idiopática submetidos à artrodese: estudo piloto. Fisioterapia em Movimento, 25(1), 165-73. https://doi.org/10.1590/S0103-51502012000100016.

Santos, M. M., Silva, M. P. C., Sanada, L. S., \& Alves, C. R. J. (2009). Análise postural fotogramétrica de crianças saudáveis de 7 a 10 anos: confiabilidade interexaminadores. Brazilian Journal of Physical Therapy, 13(4), 350-5. Epub August 28, 2009.https://doi.org/10.1590/S1413-35552009005000047.

Turtelli, C. M., (2002). Manifestações Radiológicas da Mucopolissacaridose Tipo VI. RadiolBras, 35(5), 311-4.

Valayannopoulos, V., Nicely, H., \& Harmatz, P. (2010) Mucopolysaccharidosis VI. Orphanet J Rare Dis 5, 5. https://doi.org/10.1186/1750-1172-5-5.

Vieira, T., Schwartz, I., Muñoz, V., Pinto, L., Steiner, C., Ribeiro, M., Boy, R., Ferraz, V., de Paula, A., Kim, C., Acosta, A., \& Giugliani, R. (2008). Mucopolysaccharidoses in Brazil: what happens from birth to biochemical diagnosis? American journal of medical genetics. Part A, 146(13), 1741-7. https://doi.org/10.1002/ajmg.a.32320. 